\title{
28 Research Square \\ ecRESCUE: a novel ecDHFR-regulated RESCUE system with reduced off-target activity
}

\section{Yihan Wang \\ Guo Li \\ Xiangyang Li \\ Yuzhe Wang \\ Xingxu Huang \\ Xiaoxiang $\mathrm{Hu}$ \\ Jianen Gao}

\section{Video Byte}

Keywords: dihydrofolate reductase destabilization domain, DHFR, ecDHFR, destabilization domain, DD, RNA base editing, base editor, A-to-I editing, C-to-U editing, REPAIR, RESCUE, dRanCas13b, ADAR2, trimethoprim, TMP, inducible RNA editing, off-target, SNPs, inducible stabilization, Cell Communication and Signaling

Posted Date: October 13th, 2021

DOI: https://doi.org/10.21203/rs.3.rs-966270/v1

License: (c) (i) This work is licensed under a Creative Commons Attribution 4.0 International License. Read Full License 


\section{Abstract}

RNA base-editing tools enable scientists to study gene function and develop treatments for genetic diseases. The pioneering tool REPAIR changes A bases into I bases, while the newer RESCUE system changes $\mathrm{A}$ and $\mathrm{C}$ bases into I and $\mathrm{U}$ bases, respectively. However, both systems tend to edit unintended, or off-target, sites in addition to desired sites. To enable more precise RNA editing, researchers recently designed a new system, ecRESCUE, by fusing the destabilization domain (DD) of the E. coli protein ecDHFR to the RESCUE system. The DD degrades the two RESCUE proteins (dRanCas13b and ADAR2) unless the drug TMP is present, making the system drug-inducible. In vitro, ecRESCUE was just as effective as RESCUE in the presence of TMP, but ecRESCUE-mediated editing was greatly reduced in the absence of TMP. When 3 specific sites were targeted, TMP-induced ecRESCUE made many fewer offtarget edits than RESCUE. Although ecRESCUE might need to be refined to further reduce or eliminate the remaining off-target effects, the results suggest that this new system is a promising improvement of the previous system and can achieve RNA base editing for gene function research with fewer unintended consequences. 